\title{
ON THE INVERSION OF THE LAPLACE TRANSFORM FOR RESOLVENT FAMILIES IN $U M D$ SPACES
}

\author{
IOANA CIORANESCU AND CARLOS LIZAMA
}

\begin{abstract}
We analize the inversion of the Laplace transform in $U M D$ - spaces for resolvent families associated to an integral Volterra equation of convolution type.
\end{abstract}

\section{INTRODUCTION}

Let $X$ be a complex Banach space. We consider the following Volterra equation of convolution type:

$$
u(t)=f(t)+\int_{0}^{t} a(t-s) A u(s) d s, \quad t \geq 0
$$

where $A$ is a closed linear unbounded operator with domain $D(A)$ densely defined on $X, a \in L_{\text {loc }}^{1}\left(\mathbb{R}_{+}\right)$is a scalar kernel, and $f \in L^{1}\left(\mathbb{R}_{+} ; X\right)$. We recall from [12] that a family $\{S(t)\}_{t \geq 0}$ of bounded and linear operators defined in $X$ is said to be a resolvent family for (1) if the following conditions are satisfied:

(R1) $S(t)$ is strongly continuous on $\mathbb{R}_{+}$and $R(0)=I$;

(R2) $S(t) x \in D(A)$ and $A S(t) x=S(t) A x$ for all $x \in D(A)$ and $t \geq 0$;

(R3) The resolvent equation holds

$$
S(t) x=x+\int_{0}^{t} a(t-s) S(s) A x d s
$$

for all $x \in D(A)$ and $t \geq 0$.

The notion of resolvent family is a natural extension of the concepts of a $C_{0}$-semigroup and a cosine operator function (obtained for $a(t) \equiv 1$ and $a(t) \equiv t$ respectively). The existence of a resolvent family allows one to find the solution for the equation (1). Several properties of resolvent families has been discussed in [8], [9],[11],[12],[4],[3].

In this paper we examine the convergence of the inverse Laplace transform for a resolvent family in a Banach space $X$.

In the sequel we always assume the existence of a resolvent $\{S(t)\}_{t \geq 0}$ for (1) which is in addition of type $\left(M, \omega_{0}\right)$, i.e. there are constants $M>0$ and $\omega_{0} \in \mathbb{R}_{+}$such that

$$
\|S(t)\| \leq M e^{\omega_{0} t} \text { for all } t \geq 0 .
$$

1991 Mathematics Subject Classification. Primary 45N05; Secondary 45A05, 47D99.

The first author is supported by FONDECYT Grant \#7010675.

The second author is supported by FONDECYT Grant \#1010675. 
Also, we assume the existence of the Laplace transform of $a(t)$, denoted $\hat{a}(\lambda)$, for all $\operatorname{Re} \lambda>\omega_{0}$. Under these conditions, the generation theorem for resolvent families (see [12], Theorem 1.3) give us the following

(H1) $\hat{a}(\lambda) \neq 0$ and $\frac{1}{\hat{a}(\lambda)} \in \rho(A)$ for all $\operatorname{Re} \lambda>\omega_{0}$;

(H2) $H(\lambda):=(\lambda-\lambda \hat{a}(\lambda) A)^{-1}$ satisfies

$$
H(\lambda)=\hat{S}(\lambda)=\int_{0}^{\infty} e^{-\lambda t} S(t) d t, \text { for all } \operatorname{Re} \lambda>\omega_{0}
$$

Conversely, one may express the resolvent $\{S(t)\}_{t \geq 0}$ in terms of the Laplace transform $H(\lambda)$ by different formulas. For instance, by means of the PostWidder inversion formula (see [10], Theorem 2.1) or by means of the complex inversion formula of the Laplace transform obtaining

$$
S(t) x=\frac{1}{2 \pi i} \int_{\omega-i \infty}^{\omega+i \infty} e^{\lambda t} H(\lambda) x d \lambda
$$

for all $t>0, \omega>\omega_{0}$ and $x \in D(A)$ (see Proposition 2).

Our main result in this paper establish that in $U M D$ spaces the formula (4) holds for all $x \in X$. We remark that in the particular case of $a(t) \equiv 1$ (i.e. $S(t)$ is a $C_{0}$-semigroup) we recover a result of A. Driouich and O. ElMennaoui [6] Theorem 1 (see also [2] Theorem 3.12.2), in fact, the proof of our main result is very much inspired by the proof of Driouich and ElMannaoui for the semigroup case. However, due to the more complicated structure of the Laplace transform $H(\lambda)$ the argument involved are more delicate and differ from those employed in [6]. We also observe that in the case of $a(t) \equiv t$, we recover the inversion formula for cosine operator functions in $U M D$ spaces due to I. Cioranescu and V. Keyantuo [5]. Let us recall that a Banach space $X$ is called $U M D$ if the Hilbert transform $\mathcal{H}$ defined in the Schwartz space $\mathcal{S}(\mathbb{R}, X)$ by

$$
\mathcal{H} f(t):=\lim _{\epsilon \rightarrow 0^{+}} \frac{1}{\pi} \int_{|t-s| \geq \epsilon} \frac{f(s)}{t-s} d s
$$

extends to a bounded linear operator on $L^{p}(\mathbb{R} ; X)$ for some $p \in(1, \infty)$ (or, equivalently, for all $p \in(1, \infty)$, see [1]). Note, that every $U M D$ space is reflexive and its dual is also a $U M D$ space.

In the following, we will suppose that $a \in L_{l o c}^{1}\left(\mathbb{R}_{+}\right)$is of subexponential growth, that is $\int_{0}^{\infty} e^{-\epsilon t}|a(t)| d t<\infty$, for all $\epsilon>0$. Under this condition, the Laplace transform, $\hat{a}(\lambda)$, exists for all $\operatorname{Re} \lambda>0$. Also we recall [12] that $a(t)$ is called $k$-regular $(k \in \mathbb{N})$, if there is a constant $c>0$ such that

$$
\left|\lambda^{n} \hat{a}^{(n)}(\lambda)\right| \leq c|\hat{a}(\lambda)|
$$

for all $\operatorname{Re} \lambda>0$ and $1 \leq n \leq k$. Observe that if $a \in L_{\text {loc }}^{1}\left(\mathbb{R}_{+}\right)$is of subexponential growth and 1-regular, then $\hat{a}(i s):=\lim _{\lambda \rightarrow i s} \hat{a}(\lambda)$ exists for all $s \neq 0$. Moreover, $\hat{a}(\lambda) \neq 0$ for all $\operatorname{Re} \lambda \geq 0, \lambda \neq 0$ (see [12], Lemma 8.1). We will need the following result from [11]. 
Lemma 1. Let $a \in L_{l o c}^{1}\left(\mathbb{R}_{+}\right)$be Laplace transformable and suppose that

$$
H(\lambda)=(\lambda-\lambda \hat{a}(\lambda) A)^{-1}
$$

exists for all Re $\lambda>0$. Then,

$$
H^{\prime}(\lambda)=f(\lambda) H(\lambda)+g(\lambda) H(\lambda)^{2},
$$

where $f(\lambda)=-\left(\frac{1}{\lambda}+\frac{\hat{a}^{\prime}(\lambda)}{\hat{a}(\lambda)}\right)$ and $g(\lambda)=\lambda \frac{\hat{a}^{\prime}(\lambda)}{\hat{a}(\lambda)}$ for all $\operatorname{Re} \lambda>0$.

With the notations of Lemma 1, we give the following

Lemma 2. Let $a(t)$ be 3-regular; then there is a bounded function $b \in$ $C^{1}\left(\mathbb{R}_{+}\right)$such that $\hat{b}(\lambda)=f(\lambda)$ for all $\operatorname{Re} \lambda>0$.

Proof. Since $a$ is 1-regular we have $|f(\lambda)| \leq \frac{M}{\mid \lambda} \mid$ for all $\operatorname{Re} \lambda>0$. On the other hand $f^{\prime}(\lambda)=-\left(-\frac{1}{\lambda^{2}}+\frac{\hat{a}^{\prime \prime}(\lambda) \hat{a}(\lambda)-\hat{a}^{\prime}(\lambda)^{2}}{\hat{a}(\lambda)^{2}}\right)$ implies, $\left|f^{\prime}(\lambda)\right| \leq \frac{M}{|\lambda|^{2}}$ for all $\operatorname{Re} \lambda>0$, because $a$ is 2-regular. Now $f^{\prime \prime}(\lambda)=-\frac{2}{\lambda^{3}}-\frac{\hat{a}^{\prime \prime \prime}(\lambda)}{\hat{a}(\lambda)}+\frac{\hat{a}^{\prime \prime}(\lambda)}{\hat{a}(\lambda)} \cdot \frac{\hat{a}^{\prime}(\lambda)}{\hat{a}(\lambda)}-$ $2 \frac{\hat{a}^{\prime}(\lambda)}{\hat{a}(\lambda)} \cdot\left(\frac{\hat{a}^{\prime \prime}(\lambda) \hat{a}(\lambda)-\hat{a}^{\prime}(\lambda)^{2}}{\hat{a}(\lambda)^{2}}\right)$ and as $a$ is 3-regular we obtain $\left|f^{\prime \prime}(\lambda)\right| \leq \frac{M}{|\lambda|^{3}}$ for all $\operatorname{Re} \lambda>0$. In short we have that $f(\lambda)$ satisfies

$$
\left|\lambda^{n+1} f^{(n)}(\lambda)\right| \leq M \text { for all } \operatorname{Re} \lambda>0 \text { and } n=0,1,2 .
$$

Hence, by Theorem 0.4 in [12] we obtain that there is a bounded function $b \in C^{1}\left(\mathbb{R}_{+}\right)$such that $\hat{b}(\lambda)=f(\lambda)$ for all $\operatorname{Re} \lambda>0$.

We also need the following lemma. For details we refer to the monograph of J. Prüss ( [12], Lemma 10.1).

Lemma 3. Suppose $c$ is a locally analytic function on $\mathbb{C}_{+}^{\infty}$. Then there is a function $k \in L^{1}\left(\mathbb{R}_{+}\right)$such that

$$
c(t)=c(\infty)+\hat{k}(\lambda),
$$

for all $\lambda \in \mathbb{C}_{+}^{\infty}$.

2. $L^{p}$ - ESTIMATES FOR THE INVERSiON OF THE LAPLACE TRANSFORM IN $U M D$ SPACES

For $j=1,2$, let $\left\{F_{j}(t)\right\}_{t \geq 0} \subseteq \mathbf{B}(X)$ be strongly continuous and of type $\left(M, \omega_{0}\right)$. Let

$$
\hat{F}_{j}(z)=\int_{0}^{\infty} e^{-z t} F_{j}(t) d t, \quad \operatorname{Re} z>\omega_{0}
$$

be the Laplace transform. Then, for $\omega>\omega_{0}$, we have $\hat{F}_{j}(\omega+i \lambda) \in \mathbf{B}(X)$ and $\hat{F}_{j}(\omega+i \lambda)$ are strongly continuous for $\lambda \in \mathbb{R}$; moreover

$$
\sup _{\lambda \in \mathbb{R}}\left\|\hat{F}_{j}(\omega+i \lambda)\right\|<\infty \text { and } \lim _{|\lambda| \rightarrow \infty} \hat{F}_{j}(\omega+i \lambda) x=0 \quad(x \in X) .
$$

Denote for $t \in \mathbb{R}, r<r^{\prime}$ and $x \in X$ 


$$
\begin{gathered}
I_{1}\left(t, r, r^{\prime}\right) x=\frac{1}{2 \pi} \int_{r}^{r^{\prime}} e^{i \lambda t} \hat{F}_{1}(\omega+i \lambda) x d \lambda \\
I_{2}\left(t, r, r^{\prime}\right) x=\frac{1}{2 \pi} \int_{r}^{r^{\prime}} e^{i \lambda t} \hat{F}_{1}(\omega+i \lambda) \hat{F}_{2}(\omega+i \lambda) x d \lambda .
\end{gathered}
$$

Lemma 4. We have, for all $x \in X$ :

$$
\begin{aligned}
I_{2}\left(t, r, r^{\prime}\right) x & =\int_{0}^{\infty} I_{1}\left(t-s, r, r^{\prime}\right) e^{-\omega s} F_{2}(s) x d s \\
I_{1}\left(t, r, r^{\prime}\right) x & =\frac{1}{2 i} e^{i t r^{\prime}} \mathcal{H}\left(e^{-i r^{\prime} \cdot} e^{-\omega \cdot} \chi_{[0, \infty)}(\cdot) F_{1}(\cdot) x\right)(t) \\
& -\frac{1}{2 i} e^{i t r} \mathcal{H}\left(e^{-i r \cdot} e^{-\omega \cdot} \chi_{[0, \infty)}(\cdot) F_{1}(\cdot) x\right)(t)
\end{aligned}
$$

where $\mathcal{H}$ is the Hilbert transform and $\chi_{[0, \infty)}(\cdot)$ denotes the characteristic function.

\section{Proof.}

$$
\begin{aligned}
I_{2}\left(t, r, r^{\prime}\right) x & =\frac{1}{2 \pi} \int_{r}^{r^{\prime}} e^{i \lambda t} \hat{F}_{1}(\omega+i \lambda)\left[\int_{0}^{\infty} e^{-(\omega+i \lambda) s} F_{2}(s) x d s\right] d \lambda \\
& =\frac{1}{2 \pi} \int_{0}^{\infty}\left[\int_{r}^{r^{\prime}} e^{i \lambda(t-s)} \hat{F}_{1}(\omega+i \lambda) d \lambda\right] e^{-\omega s} F_{2}(s) x d s \\
& =\frac{1}{2 \pi} \int_{0}^{\infty} I_{1}\left(t-s, r, r^{\prime}\right) e^{-\omega s} F_{2}(s) x d s
\end{aligned}
$$

where we used the fact that the function $(s, \lambda) \rightarrow \| e^{i \lambda(t-s)} e^{-\omega s} \hat{F}_{1}(\omega+$ $i \lambda) F_{2}(s) x \|$ belongs to $L^{1}\left(\mathbb{R}_{+} \times\left[r^{\prime}, r\right]\right)$.

We shall prove further the second formula. Note first that the function $(s, \lambda) \rightarrow\left\|e^{i \lambda(t-s)} e^{-\omega s} F_{1}(s) x\right\| \in L^{1}\left(\mathbb{R}_{+} \times\left[r^{\prime}, r\right]\right)$, consequently we have:

$$
\begin{aligned}
I_{1}\left(t, r, r^{\prime}\right) x & =\frac{1}{2 \pi} \int_{r}^{r^{\prime}} e^{i \lambda t}\left[\int_{0}^{\infty} e^{-(\omega+i \lambda) s} F_{1}(s) x d s\right] d \lambda \\
& =\frac{1}{2 \pi} \int_{0}^{\infty}\left[\int_{r}^{r^{\prime}} e^{i \lambda(t-s)} d \lambda\right] e^{-\omega s} F_{1}(s) x d s \\
& =\frac{1}{2 \pi i} p \cdot v \cdot \int_{0}^{\infty} \frac{e^{i(t-s) r^{\prime}}-e^{i(t-s) r}}{t-s} e^{-\omega s} F_{1}(s) x d s \\
& =\frac{e^{i t r^{\prime}}}{2 \pi i} p \cdot v \cdot \int_{0}^{\infty} \frac{e^{-i s r^{\prime}} e^{-\omega s}}{t-s} F_{1}(s) x d s-\frac{e^{i t r}}{2 \pi i} p \cdot v \cdot \int_{0}^{\infty} \frac{e^{-i s r} e^{-\omega s}}{t-s} F_{1}(s) x d s \\
& =\frac{e^{i t r^{\prime}}}{2 i} \mathcal{H}\left(e^{-i r^{\prime} \cdot} e^{-\omega \cdot} \chi_{[0, \infty)}(\cdot) F_{1}(\cdot) x\right)(t) \\
& -\frac{e^{i t r}}{2 i} \mathcal{H}\left(e^{-i r \cdot} e^{-\omega \cdot} \chi_{[0, \infty)}(\cdot) F_{1}(\cdot) x\right)(t)
\end{aligned}
$$


Proposition 1. Let $X$ be an $U M D$ space and $p \in(1, \infty)$; then there exists a constant $C>0$ such that

$$
\begin{array}{ll}
(c) & \left\|I_{1}\left(\cdot, r, r^{\prime}\right) x\right\|_{L^{p}(\mathbb{R}, X)} \leq C\|x\|, \\
(d) & \left\|I_{2}\left(\cdot, r, r^{\prime}\right) x\right\|_{L^{\infty}(\mathbb{R}, X)} \leq C\|x\|,
\end{array}
$$

for all $x \in X$ and $r<r^{\prime}$.

Proof. Using (b) we obtain

$$
\begin{aligned}
\left\|2 I_{1}\left(\cdot, r, r^{\prime}\right) x\right\|_{L^{p}(\mathbb{R}, X)} & \leq \| \mathcal{H}\left(e^{-i r^{\prime} \cdot} e^{-\omega \cdot} \chi_{[0, \infty)}(\cdot) F_{1}(\cdot) x \|_{L^{p}(\mathbb{R}, X)}\right. \\
& +\| \mathcal{H}\left(e^{-i r \cdot} e^{-\omega \cdot} \chi_{[0, \infty)}(\cdot) F_{1}(\cdot) x \|_{L^{p}(\mathbb{R}, X)}\right. \\
& \leq 2 C_{1}\left\|e^{-\omega \cdot} \chi_{[0, \infty)}(\cdot) F_{1}(\cdot) x\right\|_{L^{p}(\mathbb{R}, X)} \\
& \leq 2 C_{1} M\left(\frac{1}{\left(\omega-\omega_{0}\right) p}\right)^{1 / p}\|x\| .
\end{aligned}
$$

where $C_{1}$ is the norm of the Hilbert transform in $L^{p}(\mathbb{R}, X)$. Consider further $x \in X$ and $x^{*} \in X^{*}$; we have by (a)

$$
\begin{aligned}
\left|<x^{*}, I_{2}\left(t, r, r^{\prime}\right) x>\right| & =\left|\int_{0}^{\infty}<x^{*}, I_{1}\left(t-s, r, r^{\prime}\right) e^{-\omega s} F_{2}(s) x>d s\right| \\
& =\left|\int_{0}^{\infty}<I_{1}^{*}\left(t-s, r, r^{\prime}\right) x^{*}, e^{-\omega s} F_{2}(s) x>d s\right| \\
& \leq \int_{0}^{\infty}\left\|e^{-\omega s} F_{2}(s) x\right\| \| I_{1}^{*}\left(t-s, r, r^{\prime}\right) x^{*}|| d s \\
& \leq\left\|I_{1}^{*}\left(\cdot, r, r^{\prime}\right) x^{*}\right\|_{L^{p}(\mathbb{R}, X)}\left\|e^{-\omega \cdot} \chi_{[0, \infty)}(\cdot) F_{2}(\cdot) x\right\|_{L^{q}(\mathbb{R}, X)}
\end{aligned}
$$

where $\frac{1}{p}+\frac{1}{q}=1$. Since $X^{*}$ is also a $U M D$ space, we can use (c) to estimate $\left\|I_{1}^{*}\left(\cdot, r, r^{\prime}\right) x^{*}\right\|$ and obtain that there exists a constant $C_{2}>0$ such that

$$
\left|<x^{*}, I_{2}\left(t, r, r^{\prime}\right) x>\right| \leq C_{2}|| x^{*}|||| x||
$$

and thus (d) holds.

\section{MAIN RESUlT}

We start our considerations with the following inversion result in general Banach spaces.

Lemma 5. Let $\{S(t)\}_{t \geq 0}$ be a strongly continuous family of type $\left(M, \omega_{0}\right)$ and let $b \in C^{1}\left(\mathbb{R}_{+}\right)$be of type $\left(K, \omega_{0}\right)$; then

$$
(b * S)(t) x=\lim _{r^{\prime} \rightarrow \infty \rightarrow-\infty} \frac{1}{2 \pi} \int_{r}^{r^{\prime}} e^{(\omega+i \lambda) t} \widehat{(b * S)}(\omega+i \lambda) x d \lambda,
$$

for each $\omega>\omega_{0}$ and all $x \in X$. Moreover, the convergence is uniform on $t$ for any compact interval of $(0, \infty)$

Proof. By Theorem 6.3.1 in [7] we have for all $x \in X$ and $\omega>\omega_{0}$

$$
\int_{0}^{t}\left(b^{\prime} * S\right)(s) x d s=\frac{1}{2 \pi i} \int_{\omega-i \infty}^{\omega+i \infty} e^{\lambda t}\left(\widehat{b^{\prime} * S}\right)(\lambda) x \frac{d \lambda}{\lambda}
$$


and

$$
\int_{0}^{t} S(s) x d s=\frac{1}{2 \pi i} \int_{\omega-i \infty}^{\omega+i \infty} e^{\lambda t} \hat{S}(\lambda) x \frac{d \lambda}{\lambda}
$$

where the integrals are convergent uniformly with respect to $t$ in any compact interval of $(0, \infty)$. We have:

$$
\left(b^{\prime} * S\right)(s) x=(b * S)^{\prime}(s) x-b(0) S(s) x, \quad s \in \mathbb{R}_{+}
$$

and

$$
\left.\widehat{\left(b^{\prime} * S\right.}\right)(\lambda) x=\lambda \widehat{(b * S)}(\lambda) x-b(0) \hat{S}(\lambda) x, \quad \lambda>\omega_{0}
$$

so that (5) yields:

$$
\begin{aligned}
(b * S)(t) x-b(0) \int_{0}^{t} S(s) x d s & =\frac{1}{2 \pi i} \int_{\omega-i \infty}^{\omega+i \infty} e^{\lambda t} \widehat{(b * S)}(\lambda) x d \lambda \\
& -b(0) \frac{1}{2 \pi i} \int_{\omega-i \infty}^{\omega+i \infty} e^{\lambda t} \hat{S}(\lambda) x \frac{d \lambda}{\lambda} .
\end{aligned}
$$

Using (6) in the above equality we obtain

$$
(b * S)(t) x=\frac{1}{2 \pi i} \int_{\omega-i \infty}^{\omega+i \infty} e^{\lambda t} \widehat{(b * S)}(\lambda) x d \lambda
$$

where the convergence of the integral is uniform with respect to $t$ in any compact interval of $(0, \infty)$.

Proposition 2. Let $\{S(t)\}_{t \geq 0}$ be a resolvent family of type $\left(M, \omega_{0}\right)$ for the equation (1) and let $a \in C^{1}\left(\overline{\mathbb{R}}_{+}\right)$be of type $\left(K, \omega_{0}\right)$; then for each $x \in D(A)$ and $\omega>\omega_{0}$ we have

$$
S(t) x=\lim _{r \rightarrow-\infty} \frac{1}{r^{\prime} \rightarrow \infty} \int_{r}^{r^{\prime}} e^{(\omega+i \lambda) t} H(\omega+i \lambda) x d \lambda .
$$

where the convergence is uniform on $t$ for any compact interval of $(0, \infty)$.

Proof. For each $x \in D(A)$ we have by Lemma 5 and (2)

$$
\begin{aligned}
(a * S)(t) A x & =\lim _{r \rightarrow-\infty} \frac{1}{r^{\prime} \rightarrow \infty} \int_{r}^{r^{\prime}} e^{(\omega+i \lambda) t} \widehat{(a * S)}(\omega+i \lambda) A x d \lambda \\
& =\lim _{r \rightarrow-\infty} \frac{1}{r^{\prime} \rightarrow \infty} \int_{r}^{r^{\prime}} e^{(\omega+i \lambda) t}\left(\hat{S}(\omega+i \lambda) x-\frac{x}{\omega+i \lambda}\right) d \lambda \\
& =\lim _{r \rightarrow-\infty} r_{r^{\prime} \rightarrow \infty} \frac{1}{2 \pi} \int_{r}^{r^{\prime}} e^{(\omega+i \lambda) t} H(\omega+i \lambda) x d \lambda \\
& -\lim _{r \rightarrow-\infty} r_{r^{\prime} \rightarrow \infty} \frac{1}{2 \pi} \int_{r}^{r^{\prime}} e^{(\omega+i \lambda) t} \frac{x}{\omega+i \lambda} d \lambda \\
& =\lim _{r \rightarrow-\infty} \frac{1}{r^{\prime} \rightarrow \infty} \int_{r}^{r^{\prime}} e^{(\omega+i \lambda) t} H(\omega+i \lambda) x d \lambda-x
\end{aligned}
$$

Hence, the resolvent equation (2) implies the assertion. 
We can give now our main result.

Theorem 1. Let $\{S(t)\}_{t \geq 0}$ be a resolvent family of type $\left(M, \omega_{0}\right)$ for the equation (1), defined in a UMD space $X$; suppose that $a(t)$ is 3-regular, $\lambda \frac{\hat{a}(\lambda)^{\prime}}{\hat{a}(\lambda)}$ is locally analytic, and $|\hat{a}(\lambda)| \leq \frac{C}{|\lambda|}$ for all $|\lambda|>1$, then we have for all $x \in X$,

$$
S(t) x=\frac{1}{2 \pi} \int_{-\infty}^{\infty} e^{(\omega+i \lambda) t} H(\omega+i \lambda) x d \lambda, \quad t>0, \omega>\omega_{0}
$$

where the convergence is uniform for $t$ in compact intervals of $(0, \infty)$.

Proof. Let $r<0<r^{\prime}, t>0, x \in X$ and consider

$$
I\left(t, r, r^{\prime}\right) x=\frac{1}{2 \pi} \int_{r}^{r^{\prime}} e^{i \lambda t} \hat{S}(\omega+i \lambda) x d \lambda .
$$

An integration by parts yields:

$$
\begin{aligned}
I\left(t, r, r^{\prime}\right) x & =\frac{1}{2 i \pi t}\left(e^{i t r^{\prime}} \hat{S}\left(\omega+i r^{\prime}\right) x-e^{i t r} \hat{S}(\omega+i r) x\right) \\
& -\frac{1}{2 i \pi t} \int_{r}^{r^{\prime}} e^{i \lambda t} \hat{S}(\omega+i \lambda)^{\prime} x d \lambda .
\end{aligned}
$$

Since $\lim _{r \rightarrow-\infty} \hat{S}(\omega+i r) x=\lim _{r^{\prime} \rightarrow \infty} \hat{S}\left(\omega+i r^{\prime}\right) x=0$, in order to prove that $\lim I\left(t, r, r^{\prime}\right)$ exists as $r^{\prime} \rightarrow \infty$ and $r \rightarrow-\infty$, we only have to prove that $\lim \int_{r}^{r^{\prime}} e^{i \lambda t} \hat{S}(\omega+i \lambda)^{\prime} x d \lambda$ exists as $r^{\prime} \rightarrow \infty$ and $r \rightarrow-\infty$. Since $a$ is 3-regular we have by Lemma 1

$$
\hat{S}(\omega+i \lambda)^{\prime}=f(\omega+i \lambda) \hat{S}(\omega+i \lambda)+g(\omega+i \lambda) \hat{S}(\omega+i \lambda)^{2}
$$

with $\sup _{\operatorname{Re} z>0}|z f(z)|<\infty$ and $\sup _{\operatorname{Rez}>0}|g(z)|<\infty$. Hence we have

$$
\begin{aligned}
\int_{r}^{r^{\prime}} e^{i \lambda t} \hat{S}(\omega+i \lambda)^{\prime} x d \lambda & =\int_{r}^{r^{\prime}} e^{i \lambda t} f(\omega+i \lambda) \hat{S}(\omega+i \lambda) x d \lambda \\
& +\int_{r}^{r^{\prime}} e^{i \lambda t} g(\omega+i \lambda) \hat{S}(\omega+i \lambda)^{2} x d \lambda .
\end{aligned}
$$

Concerning the first integral in the second part of the above equality, we have by Lemma 2 that there exist a bounded function $b \in C^{1}\left(\mathbb{R}_{+}\right)$such that $\hat{b}(\lambda)=\hat{f}(\lambda)$. Hence, by Lemma 5 the integral

$$
\int_{r}^{r^{\prime}} e^{(\omega+i \lambda) t} f(\omega+i \lambda) \hat{S}(\omega+i \lambda) x d \lambda=\int_{r}^{r^{\prime}} e^{(\omega+i \lambda) t} \widehat{(b * S)}(\omega+i \lambda) x d \lambda
$$

converges to $2 \pi(b * S)(t) x$ as $r \rightarrow-\infty$ and $r^{\prime} \rightarrow \infty$, uniformly for $t$ in compact intervals of $(0, \infty)$. Next, we will prove the convergence of the integral

$$
\int_{r}^{r^{\prime}} e^{i \lambda t} g(\omega+i \lambda) \hat{S}(\omega+i \lambda)^{2} x d \lambda
$$

We shall consider first the case $x \in D\left(A^{2}\right)$. We have:

$$
\hat{S}^{2}(\omega+i \lambda) x=\frac{x}{(\omega+i \lambda)^{2}}+2 \frac{\hat{a}(\omega+i \lambda)}{\omega+i \lambda} \hat{S}(\omega+i \lambda) A x+\hat{a}^{2}(\omega+i \lambda) \hat{S}(\omega+i \lambda)^{2} A^{2} x .
$$


Using the hypothesis, it follows that there is a constant $C>0$ such that

$$
\left\|g(\omega+i \lambda) \hat{S}^{2}(\omega+i \lambda) x\right\| \leq \frac{C}{|\omega+i \lambda|^{2}}\left(\|x\|+\|A x\|+\left\|A^{2} x\right\|\right) .
$$

Consequently, the limit

$$
\int_{r}^{r^{\prime}} e^{i \lambda t} g(\omega+i \lambda) \hat{S}(\omega+i \lambda)^{2} x d \lambda
$$

exists as $r^{\prime} \rightarrow \infty$ and $r \rightarrow-\infty$, for all $x \in D\left(A^{2}\right)$, uniformly for $t$ in any compact interval of $(0, \infty)$.

Observe further that according the hypothesis and Lemma 3, there exists a function $k \in L^{1}\left(\mathbb{R}_{+}\right)$such that

$$
g(\omega+i \lambda) \hat{S}(\omega+i \lambda)^{2} x=\hat{S}(\omega+i \lambda)[(\widehat{(k * S)})(\omega+i \lambda) x+g(\infty) \hat{S}(\omega+i \lambda)]
$$

for all $x \in X$.

Let $F_{1}(t)=S(t)$ and $F_{2}(t)=(k * S)(t)+g(\infty) S(t)$. Note that $F_{2}$ is of exponential type since $k \in L^{1}\left(\mathbb{R}_{+}\right)$and hence

$$
g(\omega+i \lambda) \hat{S}(\omega+i \lambda)^{2} x=\hat{F}_{1}(\omega+i \lambda) \hat{F}_{2}(\omega+i \lambda) x
$$

for all $x \in X$.

We can now apply the estimate (d) in Proposition 1 to obtain

$$
\left\|\int_{r}^{r^{\prime}} e^{i \lambda t} g(\omega+i \lambda) \hat{S}(\omega+i \lambda)^{2} x d \lambda\right\|_{L^{\infty}\left(\mathbb{R}_{+} ; X\right)} \leq C\|x\|,
$$

for all $x \in X$.

Since $D\left(A^{2}\right)$ is dense in $X$ the above integral converges for all $x \in X$. We conclude that

$$
\frac{1}{2 \pi} \lim _{r \rightarrow-\infty} \int_{r^{\prime} \rightarrow \infty}^{r^{\prime}} e^{(\omega+i \lambda) t} \hat{S}(\omega+i \lambda) x d \lambda=R(t) x
$$

exists for all $x \in X$, uniformly for $t$ in any compact interval of $(0, \infty)$.

On the other hand, by equation (6), we have for all $x \in X$

$$
\int_{0}^{t} S(s) x d s=\frac{1}{2 \pi} \lim _{r \rightarrow-\infty} \int_{r}^{r^{\prime} \rightarrow \infty} e^{(\omega+i \lambda) t} \hat{S}(\omega+i \lambda) x \frac{d \lambda}{\omega+i \lambda}
$$

uniformly for $t$ in any compact interval of $(0, \infty)$. By differentiation we obtain $R(t) x=S(t) x$ for all $x \in X$.

Remark The functions $a(t)=\frac{t^{\alpha}}{\Gamma(\alpha+1)}(\alpha \geq 0)$ satisfy the conditions of the above Theorem. In particular, for $\alpha=0$ and $\alpha=1$ we recover the results in [6], Theorem 1 and [5], Proposition 2.12 respectively.

\section{REFERENCES}

[1] H. Amann, Linear and Quasilinear Parabolic Problems: Abstract Linear Theory. Birkhäuser Verlag, Basel/Boston/Berlin, 1995.

[2] W.Arendt, C.J.K. Batty, M. Hieber, F. Neubrander, Vector-valued Laplace Transforms and Cauchy Problems, Monographs in Mathematics, Vol. 96, Birkhäuser Verlag, Basel/Boston/Berlin, 2001. 
[3] W.Arendt, J. Prüss, Vector-valued Tauberian theorems and asymptotic behavior of linear Volterra equations, SIAM J. Math. Anal. 23 N2 (1992), 412-448.

[4] J.-C. Chan, S.-Y. Shaw, Rates of approximation and ergodic limits of resolvent families, Arch.Math. 66 (1996), 320-330.

[5] I. Cioranescu, V. Keyantuo, On operator cosine functions in UMD spaces, Semigroup Forum, 63 (3) (2001), 429-440.

[6] A.Driouich, O. El-Mennaoui, On the inverse formula of Laplace transforms, Arch. Math. 72 (1999), 56-63.

[7] E. Hille and R.S. Phillips, Functional Analysis and Semigroups, Amer. Math. Soc. Providence, 1957.

[8] C. Lizama, On an extension of the Trotter-Kato theorem for resolvent families of operators, J. Integral Equations Appl. 2 (1990), 269-280.

[9] C. Lizama, On Volterra equations associated with a linear operator, Proc. Amer. Math. Soc. 118, No. 4 (1993), 1159-1166.

[10] C. Lizama, A representation formula for strongly continuous resolvent families J. Integral Equations Appl. 9 (1997), 321-327.

[11] C. Lizama, A characterization of uniform continuity for Volterra equations in Hilbert spaces, Proc. Amer. Math. Soc. 126, No. 12 (1998), 3581-3587.

[12] J. Prüss, Evolutionary Integral Equations and Applications, Monographs in Mathematics, Vol. 87, Birkhäuser Verlag, Basel/Boston/Berlin, 1993.

Department of Mathematics, University of Puerto Rico, P.O. Box 23355, Rio Piedras, Puerto Rico 00931

E-mail address: eciorane@rrpac.upr.clu.edu

Departamento de Matemática, Universidad de Santiago de Chile, Casilla 307-Correo 2, Santiago-Chile.

E-mail address: clizama@usach.cl 